\title{
PHASE SCAN SIGNATURE MATCHING FOR LINAC TUNING
}

\author{
T.L. OWENS, M.B. POPOVIC, E.S. McCRORY, \\ C.W. SCHMIDT and L.J. ALLEN \\ Fermi National Accelerator Laboratory, P.O. Box 500, Batavia, \\ Illinois 60510, USA
}

(Received 6 July 1993; in final form 11 August 1994)

\begin{abstract}
A conceptually simple method for linac tuning has been tested on the $200 \mathrm{MeV}$ drift-tube linear accelerator at Fermilab. The phase of a beam-induced signal in a stripline detector is measured as tank phase is scanned over approximately 360 degrees. The stripline detector is downstream of the tuned tank. The resulting phase-scan curves have unique signatures that depend upon only a few critical parameters. By matching theoretical curves of beam phase versus tank phase to measured curves, the proper values for these parameters are determined. The parameters which are varied during curve matching are the field amplitude, the relative tank-phase offset of the phase-scan curves and the velocity of the beam as it enters the tank. The actual phase of the tank fields relative to the design phase is found by noting the position of the actual tank phase along the phase-scan curve, once a curve match is obtained.
\end{abstract}

KEY WORDS: Instrumentation, linear accelerators, radio-frequency devices

\section{INTRODUCTION}

The phase-scan signature for a linear accelerator (linac) tank is a curve which represents the phase of a radio-frequency (rf) signal induced by the linac beam in a beam monitor such as a stripline detector, as a function of the phase of the electromagnetic fields within the linac tank. Accurate and detailed comparison of broad phase-scan signatures with theory was suggested at Fermilab in $1990^{1}$ as a means of accurately determining tank field amplitude, phase, and input beta. A similar idea was also proposed independently at the Los Alamos National Laboratory. ${ }^{2}$ The early reports demonstrated that characteristic features of the phase-scan curves depend in unique ways upon input beta and tank electric field. References 3-5 are more recent papers which allude to the idea.

The phase-scan signature matching technique can be used to find all tuning parameters needed to center the beam in longitudinal phase space, including tank electric field, tank phase and the beta of the beam at the entrance of the tank. Originally, phase-scan signature matching was suggested as a means of coarse-tuning in linacs, ${ }^{1,3}$ using the classical delta- $t$ procedure $^{6}$ for fine tuning. However, aspects of the delta- $t$ procedure can be somewhat 
complicated. This disadvantage has slowed general acceptance of the delta- $t$ procedure by machine operators and other accelerator workers at Fermilab. The phase-scan procedure is generally much easier to understand than the delta- $t$ procedure, and has had a better reception at Fermilab among machine operators, although much greater computational power is required to implement this technique compared to the delta- $t$ procedure.

Detailed analysis of the phase-scan signature matching technique reveals that accuracies are high enough that acceptable linac tuning is possible using only this technique. Results to be presented in Section 5, as well as results published by LANL, ${ }^{2}$ suggest that the phasescan signature matching technique may provide accuracies of a few tenths of a percent if tank and beam phases can be determined to \pm 1 degree.

Another advantage of the phase scan signature matching procedure over the classic delta- $t$ procedure is the reliability with which the procedure converges on the proper longitudinal values. For the delta- $t$ procedure to converge, it is necessary to know that the procedure has started near the correct values for the tank parameters. For the highenergy tanks of the Fermilab linac, when the delta- $t$ procedure is used and the tank input energy is assumed to equal its design value, errors in the results are compounded. ${ }^{1}$ The phase scan signature matching technique, on the other hand, makes no assumptions about any of the tank parameters. In addition, the phase-scan signature technique can be used even if the tank settings are far from design values. Also, of some relevance is that the phase-scan signature technique requires only a single beam monitor located downstream of the the tank being tuned, whereas the delta- $t$ procedure requires two beam monitors.

Because of the aforementioned advantages, the phase-scan signature matching technique has been adopted as one of the tools that will be used to tune the new Linac Upgrade at Fermilab $^{7}$ after it is installed in the coming months. Commissioning of the new linac with beam will commence late in the summer of 1993. It is planned to use the delta- $t$ procedure and spectrometer measurements in the upgraded linac to corroborate phase-scan signature matching results.

\section{PHASE-SCAN CONCEPT AND EXPERIMENTAL PROCEDURES}

Phase-scan signature matching is illustrated schematically in Figure 1. In the figure, $\operatorname{tank} N$ is the tank being tuned. All upstream tanks are turned on and all downstream tanks are turned off. The rf power for tank $N$ is initially turned off. The phase of the beam-induced rf signal (beam phase) at a downstream monitor is then recorded. This zero-power, unaccelerated beam phase reading is subtracted from all subsequent, accelerated beam phase readings. The theoretical accelerated beam phases are similarly referenced to the phase of an unaccelerated beam. The beam pickup can be immediately downstream of the tank being measured (if there are space constraints, like at the end of the linac), or, for higher accuracy, the pickup downstream of the next tank can be used.

Radio-frequency power is applied to tank $N$ and the beam-phase at the beam monitor is recorded as a function of the phase of the accelerating fields within tank $N$. Tank $N$ phase is varied over approximately 360 degrees during this procedure. The tank phase signal is derived from direct measurements of tank fields using pickup loops within the tanks. Phase detection for both the beam signal and the tank field is performed using I\&Q 


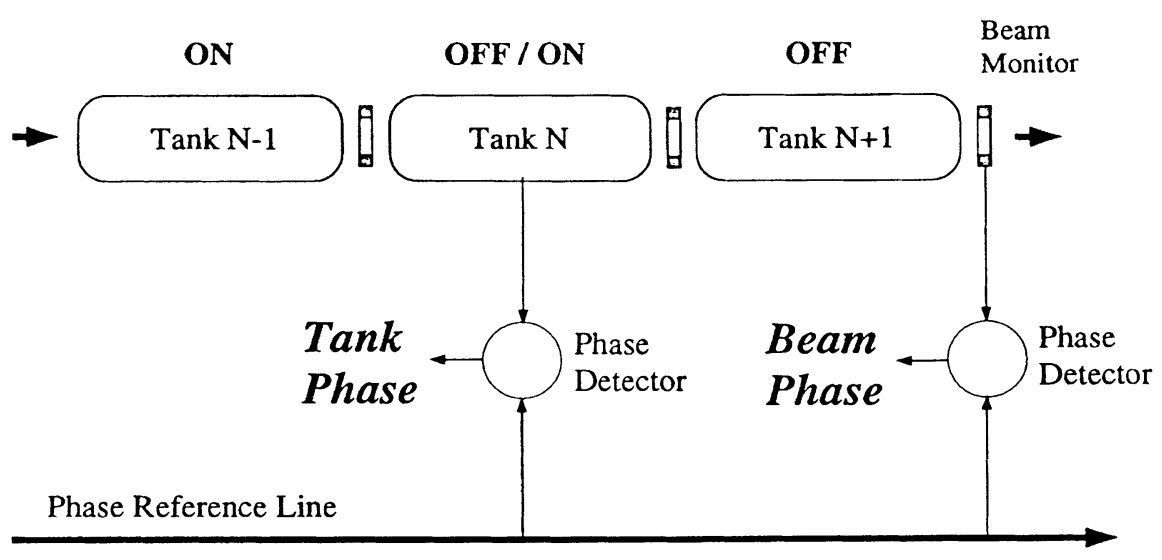

FIGURE 1: Schematic diagram of the phase-scan signature monitoring system.

demodulators. Use of I\&Q demodulators for phase detection was first suggested to us by colleagues at the Los Alamos National Laboratory. ${ }^{8}$ At Los Alamos, I\&Q demodulators are used for phase detection in their delta- $t$ system on LAMPF.

The phase detector has a measurement range of \pm 180 degrees. The beam phase can vary over a range much greater than this, making it necessary to eliminate the 360-degree ambiguities in the raw data. The data-analysis software detects large, abrupt changes in the phase which occur as the input phase goes past the extremes of the phase detector range, recording the number of these abrupt signal changes and the direction in which they occur. The number and direction of the changes determines the number and sign of 360 degree increments that will be added to the phase. Ambiguities due to periodicity in the raw data are thereby eliminated.

Tank phase is varied over approximately 360 degrees in order to make a complete comparison between theory and experiment. Also, verification can be made that the beam phase at 360 degrees of tank phase equals the beam phase at 0 degrees of tank phase. If the beam phases are equal at each extreme of tank phase, we can be sure that the software has properly tracked any 360 degree jumps in beam phase readings due to phase detector periodicity.

A special application program, designed within the framework of the Fermilab ACNET control system, has been written to control and analyze the phase scans. After raw phase detector signals have been collected and converted to beam phase and tank phase, the operator can shift the whole phase-scan curve continuously along the tank-phase axis, and in 360 degree phase increments along the beam-phase axis. This feature allows the operator to manually line up the measured phase-scan curve as closely as possible with a target curve that is superimposed on the graph of beam phase versus tank phase. The target curve is generated from the theory using design values for electric field and input beta for the tank. The movement of the phase-scan curve follows the movement of a cursor placed directly on the display of beam phase versus tank phase.

Once a reasonable curve fit to the target curve is obtained through manual movement 
of the experimental phase-scan curve, a least-squares procedure is conducted to find the theoretical curve which best matches the measured data. The program first requests that the operator select up to 50 points along the experimental phase-scan curve. The theoretical curve is fit to these points. With manual selection of points, the operator can avoid curve fitting to noisy regions or to regions where there may be beam loss. The operator is also free to select points in portions of the curves that are particularly sensitive to variations in electric field and input beta.

For least-squares curve fitting, the subroutine, MINUIT, from the CERN numerical library, is used. The parameters which are allowed to vary are; tank electric field, offset of the measured tank phase and the input beta of the beam. These three parameters are input into a subroutine which calculates the theoretical values of the beam phase at the selected points. Within MINUIT, the square root of the sum of the squares of the differences between measured beam phase and calculated beam phase is minimized as a function of the input parameters at the selected points. Once the least-squares parameters have been found, the program shifts the measured data by the calculated tank-phase offset and draws the leastsquares curve through the measured phase-scan points. The electric field, tank-phase offset and input beta derived from the least-squares fit are printed on the monitor.

Finally, a marker appears on the phase-scan graph at the position of the current tank phase setting. The marker follows any tank phase adjustments as they occur in real time. The operator can now adjust the tank phase to the appropriate position along the phase-scan curve. If the electric field and input beta equal design values, the proper tank phase setting will be zero on the phase scan curve, since all phases have been referenced to the design phase in the theory. Tank phases different from zero may be appropriate if electric field and input beta are not equal to design.

\section{PHASE-SCAN THEORY}

The principle parameter that must be calculated is the phase of the beam-induced rf signal (beam phase) at the beam monitor which is located after the tank being tuned. Beam phase is directly proportional to the time of arrival of the beam at the monitor. In this sense, the present measurements are time of flight measurements. Since it is convenient and accurate to measure phase changes at a fixed position using a single phase monitor, the phase difference between unaccelerated and accelerated beam at fixed positions will be obtained in the following calculations.

The difference between unaccelerated beam phase and accelerated beam phase is a function of beta and beam phase at the input to the tank, and to the accelerating field. This phase difference is given by,

$$
\Delta \Theta=\omega\left(\frac{D_{A B}+D_{d}}{v_{A}}-\frac{D_{d}}{v_{B}}\right)-2 \pi N_{c}-\Phi_{B}+\Phi_{A}
$$

where $D_{A B}$ is the length of the tank, $D_{d}$ is the distance from the end of the tank to the beam phase monitor, $v_{A}$ is the beam velocity at the entrance to the tank, $v_{B}$ is the beam velocity at the exit to the tank, $N_{c}$ is the number of cells within the tank, $\Phi_{B}$ is the beam phase with the rf on at the exit of the tank, and $\Phi_{A}$ is the beam phase at the entrance of the tank. Beam 
phase at the entrance and exit of the tank is referenced to the phase of the rf field within the tank.

The longitudinal dynamics used to calculate quantities on the right side of Equation 1, utilize a "thin lens" approximation. ${ }^{9}$ Using this approximation, the beam drifts freely to the center of each cell of the tank. In the center of the cell, a step change in beam velocity is calculated and the beam drifts from the center of the cell to the cell exit with the new velocity. A step change in phase, which contributes to the phase at the exit of the cell, also occurs at the center of the cell. The procedure is repeated for each cell of a tank to yield output beta and beam phase for the entire tank as a function of input beta and beam phase. The equation which govern these dynamics are the following

$$
\begin{gathered}
\Phi_{e}=\Phi_{i}+l_{1} \frac{d \Phi_{i}}{d z}+l_{2}\left(\frac{d \Phi_{i}}{d z}+\Delta \Phi\right)+\delta \Phi \\
\Delta W=\mathrm{eV} \cos \left(\Phi_{0}\right)\left[T_{o}-2 \pi\left(\frac{\beta_{g}}{\beta_{c}}-1\right) T_{p}\right] \\
\Phi_{o}=\Phi_{i}+l_{1} \frac{d \Phi_{i}}{d z}+\delta \psi \\
\beta_{c}=\beta_{i}+\frac{\mathrm{eV}}{2 \beta_{i} \gamma_{i}^{3} E_{r}}\left[T_{o} \cos \left(\Phi_{o}\right)+S_{o} \sin \left(\Phi_{o}\right)\right]
\end{gathered}
$$

where,

$$
\begin{gathered}
\frac{d \Phi_{i}}{d z}=\frac{2 \pi}{\beta_{i} \lambda} \\
\Delta \Phi=-\frac{2 \pi \Delta W}{\lambda \beta_{i}^{3} \gamma_{i}^{3} E_{r}} \\
\delta \Phi=-\frac{2 \pi \mathrm{eV} \beta_{g}}{\beta_{o}^{3} \gamma_{o}^{3} E_{r}} T_{p} \sin \left(\Phi_{o}\right) \\
\delta \psi=-\frac{\pi \mathrm{eV} \beta_{h}}{\beta_{h}^{3} \gamma_{h}^{3} E_{r}}\left[T_{p} \sin \left(\Phi_{o}\right)+S_{o} \cos \left(\Phi_{o}\right)\right] \\
\beta_{o}=\frac{\beta_{i}+\beta_{e}}{2}, \quad \beta_{h}=\frac{\beta_{i}+\beta_{c}}{2}, \quad \beta_{g}=\frac{L}{\lambda} \\
T_{o}=\frac{1}{V} \int_{-\frac{L}{2}}^{\frac{L}{2}} E(z) \cos (k z) d z \\
S_{o}=\frac{2}{V} \int_{0}^{\frac{L}{2}} E(z) \sin (k z) d z
\end{gathered}
$$




$$
\begin{gathered}
T_{p}=\frac{k}{2 \pi V} \int_{-\frac{L}{2}}^{\frac{L}{2}} z E(z) \sin (k z) d z \\
S_{p}=\frac{k}{\pi V} \int_{0}^{\frac{L}{2}} z E(z) \cos (k z) d z \\
k=\frac{2 \pi}{\beta_{g} \lambda}
\end{gathered}
$$

In the above equations, $\Phi_{e}$ is the beam phase at the exit of the cell relative to the phase of the tank rf field, $\Phi_{i}$ is the beam phase at the entrance of the cell relative to the tank if field, $\Phi_{0}$ is the beam phase at the center of the cell, $\beta_{c}$ is beta the center of the cell, $l_{1}$ is the distance from the cell entrance to the center of the cell, $l_{2}$ is the distance from the center of the cell to the end of the cell, $\Delta W$ is the beam energy change through the cell, and $V$ is the accelerating voltage across the cell. The quantity, $\delta \Phi$, is the phase correction required at the center of the cell to yield the correct exit phase. The quantity, $\delta \Psi$, is the phase correction required at the center of the cell to produce the correct central phase. The quantities, $T_{o}, S_{o}$ $T_{p}$, and $S_{p}$, are the transit time factors for the cells. The transit time factors are calculated using the electrodynamics code, SUPERFISH.

\section{PHASE SCAN SIGNATURE MATCHING EXPERIMENTS}

Figure 2 is an example of the phase-scan data that are obtained on tank 6 of the drift-tube linac at Fermilab. Tank 6 is approximately two-thirds of the way down the linear accelerator. For tank 6, design input beta is 0.45692 and design output beta is 0.49127 . The average accelerating gradient is $2.56 \mathrm{MV} / \mathrm{m}$. The distance from the end of tank 6 to the beam monitor is 16.96 meters (at the far end of tank 7).

The solid line in Figure 2 is the least-squares curve fit to the measured data. On the scale presented in Figure 2, the measured data exhibits very little noise. The fitted curve is nearly indistinguishable from the measured points. The dashed line in Figure 2 is the design curve. The deviation in the design curve from the fitted curve is adequate to clearly distinguish the two curves. Information presented in the legend of the graph expresses tank electric field and input beta for the tank as fractions of design values. From the least-squares fitting procedure, the difference between design and measured phase-scan curves in this case indicates that the tank electric field is $2.6 \%$ above the design value and the input beta is $0.18 \%$ below design.

Phase-scan signature matching was carried out for each linac tank beginning with tank 3 and ending with tank 9, which is the last tank of the linac. Plots very similar to Figure 2 were generated for each of these linac tanks. Input beta, tank field gradient, and present phase setting were found for each tank using the curve matching procedure. 


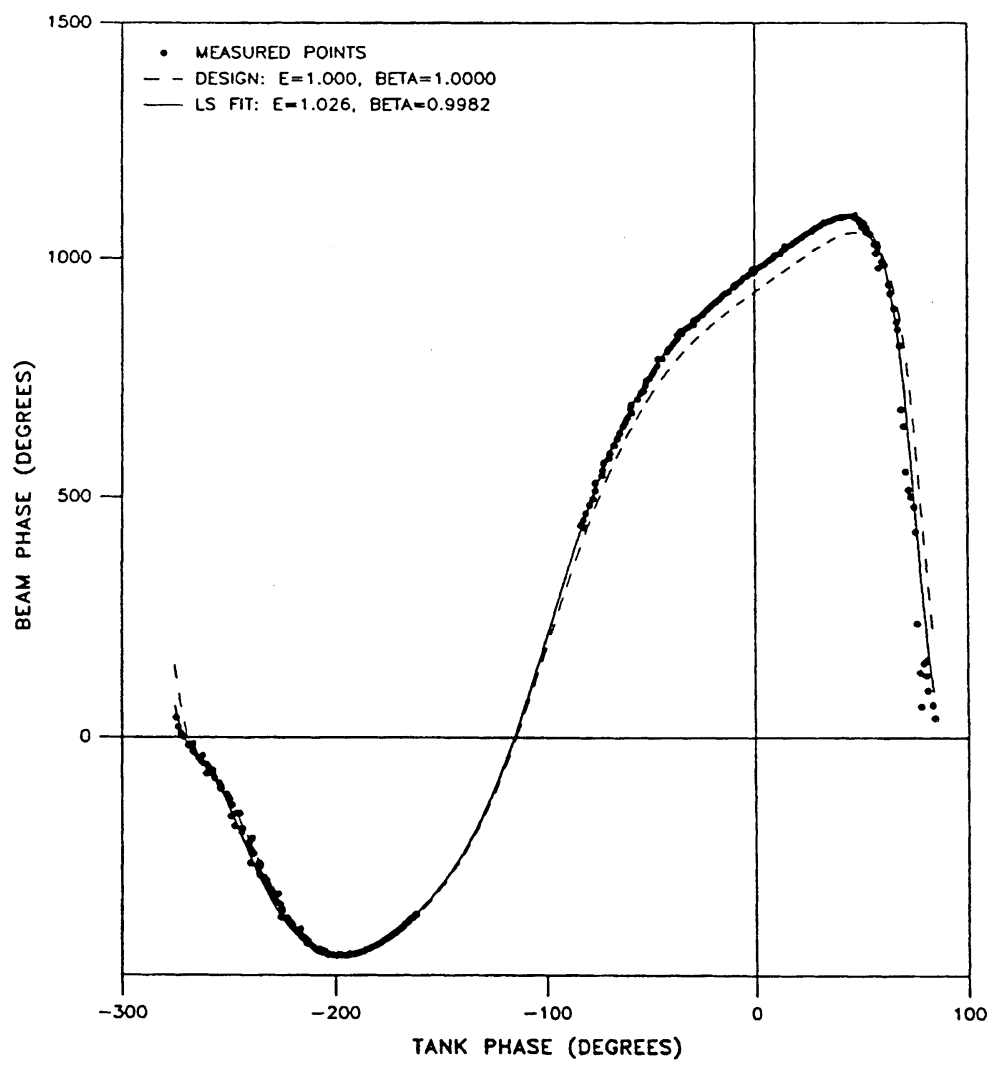

FIGURE 2: Phase-scan curves for tank 6 of the Fermilab drift-tube linac. Beam phase is measured 16.96 meters past the end of tank 6. Tank electric field (E) and beta (at input) are referenced to design values.

Table 1 presents a summary of the parameters found for each tank. Once again, the tank electric field and input beta are expressed as fractions of design values. The phase deviations indicated in Table 1 are found by noting the position of the tank phase along the phase-scan curve relative to the design phase. Table 1 demonstrates that tuning parameter deviations from design are fairly large in some of the high-energy tanks of the Fermilab linac. Of particular interest is the result for tank 9 showing that the output beta of the linac is high by $0.73 \%$. This implies that the output energy of the linac is really $204.5 \mathrm{MeV}$ instead of the design value of $200.5 \mathrm{MeV}$. This result is consistent with estimates of linac output energy based upon booster magnetic field values required to contain linac beam during injection into the booster. The tank tuning parameters, derived from the phase-scan measurements, are nominal settings that have been used for many years on the Fermilab linac. No attempt was made to correct the linac settings, since the linac has been injecting an acceptable beam into the booster synchrotron downstream of the linac and retuning the linac in this manner certainly would be quite disruptive. 
TABLE 1: Tuning parameters derived from phase-scan signature matching for Fermilab linac tanks 3-9. Output beta is calculated from tank field, phase, and input beta.

\begin{tabular}{ccccc}
\hline Tank & E Field & $\Delta$ Phase & Input $\beta$ & Output $\beta$ \\
\hline 3 & 1.001 & 2.8 & 0.9958 & 0.9970 \\
4 & 0.9724 & -4.7 & 1.000 & 1.0014 \\
5 & 0.9355 & 12.19 & 1.002 & 0.9989 \\
6 & 1.022 & 20.56 & 0.9976 & 1.0060 \\
7 & 1.014 & 20.50 & 1.006 & 1.0007 \\
8 & 0.9767 & 23.23 & 1.002 & 1.0042 \\
9 & 0.9752 & 44.65 & 1.003 & 1.0073 \\
\hline
\end{tabular}

The last column of numbers in Table 1 , labeled Output $\beta$, lists the output beta for the tank calculated from the measured tank electric field, phase offset, and input beta (parameters listed in the other three columns of Table 1). As a check of phase-scan matching results, the output beta calculated for each tank should equal the measured input beta for the downstream tank. From the numbers in Table 1, this appears to be the case to an accuracy of less than a few tenths of a percent.

\section{PHASE-SCAN ERRORS}

Simulations are used to estimate the magnitude of errors in input beta, tank field, and tank phase which can occur in the phase-scan signature matching procedure. A simulated experimental phase-scan curve is generated from theory by adding a random phase increment to the tank phase, and inputting this randomly fluctuating tank phase into the theoretical calculation of beam phase. A second random phase fluctuation is added to the calculated beam phase. The effects of random fluctuations in phase readings, which constitute the principal source of random error in the phase-scan technique, can thereby be determined. It is assumed that there are no sources of systematic error. The simulated phase-scan curves are input directly into the data acquisition program used to gather and analyze experimental phase-scans, substituting for real phase-scan measurements.

Figure 3 contains bar charts summarizing the results of a sample simulation for tank 6 of the Fermilab linac. One hundred and twenty-one separate cases were run to produce the bar charts. Seven points approximately equally spaced along the phase-scan curves were selected for the least-squares fitting procedure. Random errors in tank phase and beam phase for each run were \pm 1 degree. The tank Field for this case was set to 0.95 and the input beta to 1.002 .

Figure 3a shows the distribution of tank field amplitude errors resulting from the random phase errors. The distribution is peaked near zero with a standard deviation of $0.22 \%$. Figure $3 \mathrm{~b}$ shows the distribution of input beta error. The distribution is peaked slightly below zero 


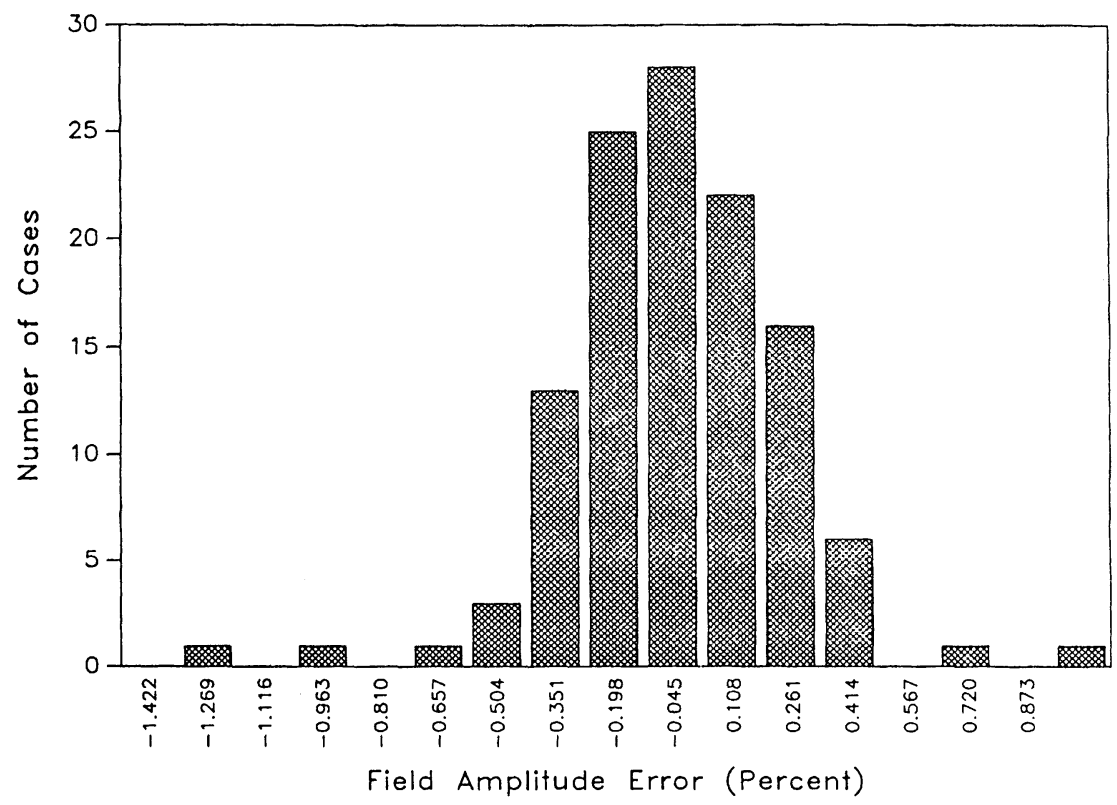

FIGURE 3a:

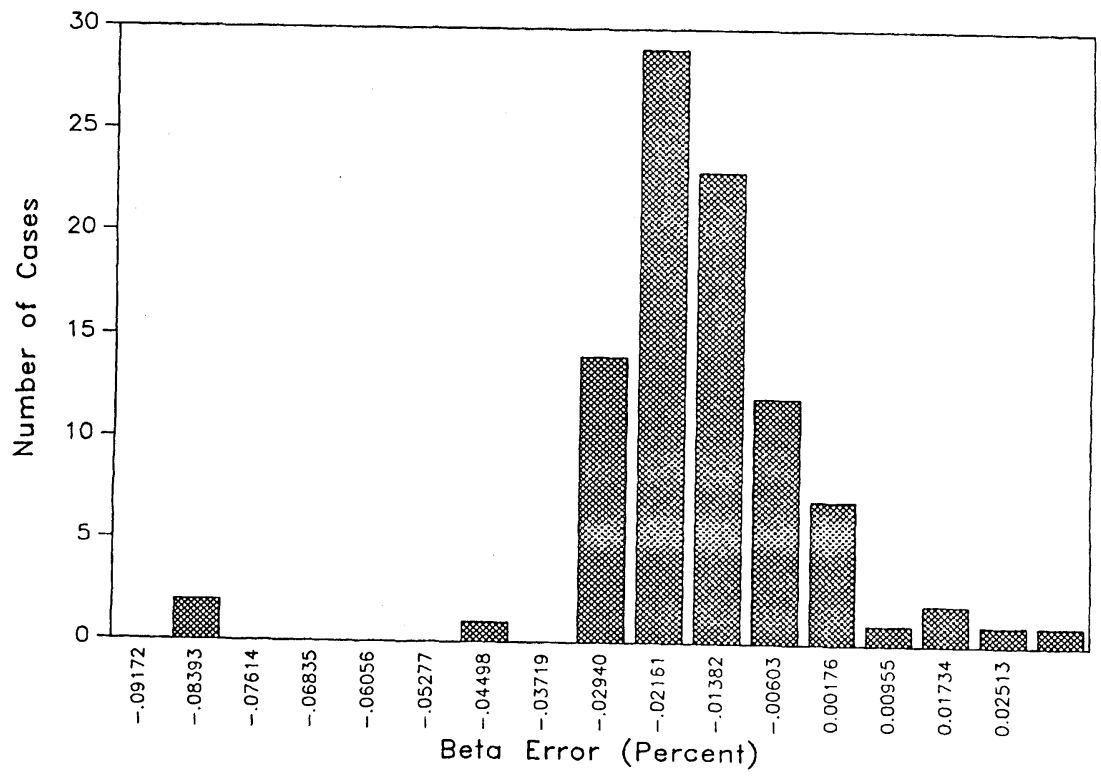

FIGI JRE 3b: 


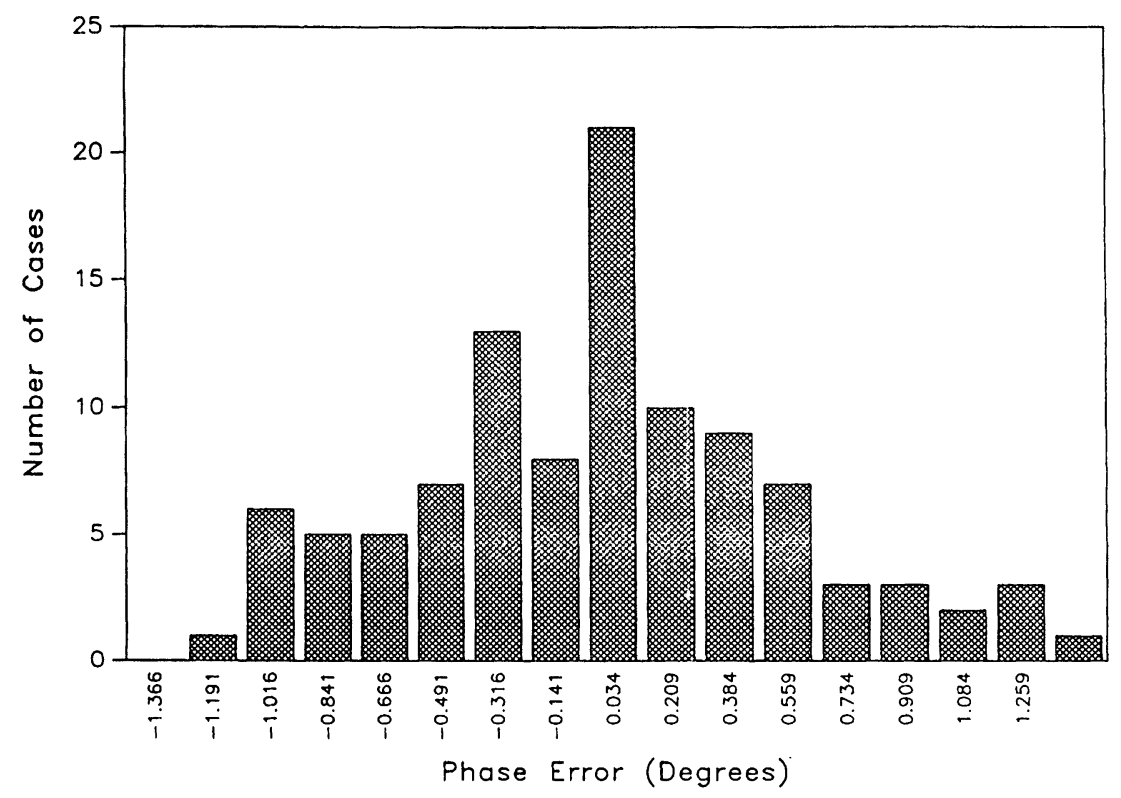

FIGURE 3c:

FIGURE 3: Distribution of errors in the phase-scan signature matching scheme for tank 6 of the Fermilab linac. Electric field in the simulation is 0.95 , and input beta is 1.002 . Tank phase and beam phase have random fluctuations of \pm 1 degree. Standard deviations are: (a) $0.22 \%$, (b) $0.011 \%$, (c) 0.43 degrees.

and the standard deviation in input beta is $0.011 \%$. Finally, Figure $3 \mathrm{c}$ shows the distribution of errors in tank phase readings calculated by the program (not the random errors input into the program). This distribution is peaked near zero with a standard deviation of 0.43 degrees.

\section{CONCLUSIONS}

The phase-scan signature matching technique is a new technique for determining the important parameters required for linac tuning. Experimental tests on the Fermilab linac have demonstrated the feasibility and advantages of the technique. It is an operationally simple method that can be easily utilized by linac machine operators. If random phase measurement errors can be kept below about one degree, the accuracy of the technique will be adequate for the Linac Upgrade at Fermilab and most other linacs, as well.

During the review process for this paper, the authors have become aware that the term "Phase Scan" has been used to describe other experimental procedures employed in the longitudinal tune-up of an ion linac. These methods are presented in the Reference. ${ }^{10}$ 


\section{REFERENCES}

1. T.L. Owens, "Phase and Amplitude Tuning Procedures for the Fermilab Linac," Fermilab Report TM-1713, Jan., 1991.

2. F.W. Guy and T.P. Wangler, "Lest-Squares Fitting Procedure for Setting RF Phase and Amplitude in DriftTube-Linac Tanks," in Conference Record of the 1991 IEEE Particle Accelerator Conference, May 6-9 (1991), pp. 3056-3058.

3. T.L. Owens and E.S. McCrory, "The Delta-T Tuneup Procedure for the Fermilab Linac Upgrade," ibid, pp. 3064-3066.

4. P.N. Ostroumov, et al., "Proton Beam Acceleration up to $160 \mathrm{MeV}$ at the Moscow Meson Factory Linac," ibid, pp. 3067-3069.

5. T.L. Owens and M.B. Popovic, "Phse-Scan Signature Matching for Linac Tuning," Fermilab Report LU186, Nov. $18,1992$.

6. K.R. Crandall, “The Delta-T Tuneup Procedure for the LAMPF 805 MHz Linac,” LANL Report LA-374MS, June, 1976.

7. C.W. Schmidt, "The Fermilab $400 \mathrm{MeV}$ Linac Upgrade," to be published in Conference Record of the 1993 Particle Accelerator Conference, May 17-20, 1991.

8. Olin Vandyck, LANL, ptivte communication.

9. B. Schnizer (1971), Part. Accl, 2, 141-147.

10. e.g., R.A. Jameson, W.E. Jule, R.S. Mills, E.D. Bush, Jr., R.L. Gluckstern, LANL Report LA-6863, March 1978. 